\title{
O uso de ducha: efeito sobre o bem-estar e a qualidade da carcaça e da carne em suínos transportados para o abate
}

\author{
[Use of shower: the effect on welfare and carcass and meat quality of pigs \\ transported to slaughter] \\ O.A. Dalla Costa ${ }^{1,4}$, T.A. Diesel ${ }^{2,4}$, M.J.R.P. Costa $^{3,4}$, F.A. Dalla Costa ${ }^{2,4}$ \\ ${ }^{1}$ Embrapa Suínos e Aves - Concórdia, SC \\ ${ }^{2}$ Aluno de pós-graduação - FCAV-Unesp - Jaboticabal, SP \\ ${ }^{3}$ FCAV-Unesp - Jaboticabal, SP \\ ${ }^{4}$ ETCO - Grupo de Estudos e Pesquisa em Etologia e Ecologia Animal
}

\begin{abstract}
RESUMO
O objetivo deste trabalho foi avaliar os efeitos do uso da ducha de água, antes e após o transporte, sobre o bem-estar dos animais e a qualidade da carne dos suínos. Foram utilizados 384 animais (fêmeas e machos castrados), frutos de cruzamento industrial e provenientes de 16 granjas de Santa Catarina/Brasil. O delineamento experimental foi em um fatorial $2 \times 4$, com duas distâncias da granja até o frigorífico (menos de $50 \mathrm{~km}$; mais de $50 \mathrm{~km}$ ) e quatro protocolos de ducha, que consistiram em: suínos não molhados na granja e no frigorífico (NMG_NMF); não molhados na granja e molhados no frigorífico (NMG_MOF); molhados na granja e não molhados no frigorífico (MOG_NMF); molhados na granja e molhados no frigorífico (MOG_MOF). Nenhum dos fatores teve influência $(\mathrm{P}>0,05)$ sobre as variáveis fisiológicas relacionadas ao estresse (cortisol e lactato sanguíneo), ao número de lesões na carcaça e às características de qualidade da carne.
\end{abstract}

Palavras-chave: suínos, spray de água, bem-estar, qualidade da carne

\begin{abstract}
This study was carried out to evaluate the effects of a shower before and after pig transportation on welfare, meat and carcass quality. We used 384 crossbreeding animals (females and castrated males), from 16 farms in Santa Catarina State, Brazil. A $2 \times 4$ factorial arrangement of treatments was employed with two distances from farm to slaughter plant (less than $50 \mathrm{~km}$ and more than $50 \mathrm{~km}$ ) and four showering protocols. These protocols were applied on pigs: no showering at farm or slaughter plant (Control), no showering at farm but showering at the plant (NMG_MOF); showering at the farm and no showering at the plant (MOG_NMF); showering at the farm and plant $\left(M O G_{-} M O F\right)$. None of the factors influenced $(P>0.05)$ physiological variables related to stress (cortisol and lactate in blood samples), number of carcass lesions and the characteristics of meat quality.
\end{abstract}

Keywords: pigs, water spray, showering, welfare, meat quality

\section{INTRODUÇÃO}

O período que antecede o abate dos suínos é considerado um dos pontos críticos de maior impacto dentro do sistema de produção (MotaRojas et al., 2011). Procedimentos inadequados durante esse manejo podem comprometer os resultados obtidos em todas as fases anteriores, interferindo diretamente sobre a saúde e o bem-

Recebido em 13 de junho de 2014

Aceito em 9 de janeiro de 2015

E-mail: osmar.dallacosta@embrapa.br estar dos animais, a qualidade da carcaça e as características físico-químicas da carne (Dalla Costa et al., 2010; Faucitano et al., 2010). Os suínos entregues ao frigorífico devem estar limpos, saudáveis, em jejum, isentos de hematomas e lesões, não estressados e em condições de se locomoverem por si só. No entanto, um dos grandes desafios é amenizar o estresse por calor, principalmente em locais quentes. 
É conhecido que, sob as condições subtropicais, o uso da água no manejo pré-abate ajuda a aliviar o estresse por calor em suínos (Gregory, 2008). Já o uso da ducha, antes ou após o transporte, como forma de promover a perda de calor dos animais no caminhão é recomendada por normativas e diretivas de vários países (Colleu e Chevillon, 1999). No Brasil, essa técnica também vem sendo utilizada por granjas e frigoríficos como forma de amenizar o estresse provocado pelo calor e reduzir as perdas durante o transporte.

Entretanto, dados sobre as consequências dessa prática para os animais e para a cadeia produtora em situações comerciais ainda não foram relatados na comunidade científica. Assim, o objetivo deste trabalho foi avaliar o efeito do uso de ducha antes e após o transporte a diferentes distâncias sobre o bem-estar, e a qualidade da carne e da carcaça de suínos em situações comerciais.

\section{MATERIAL E MÉTODOS}

O experimento foi realizado no oeste de Santa Catarina - Brasil, durante o verão de 2010. A região é caracterizada pelo clima (Cfa) subtropical, e a temperatura nos dias de coleta variou de $28^{\circ} \mathrm{C}$ a $34^{\circ} \mathrm{C}$ para a máxima e de $15^{\circ} \mathrm{C}$ a $20^{\circ} \mathrm{C}$ para a mínima.

O experimento foi desenvolvido de acordo com os princípios éticos na experimentação animal (protocolo $\mathrm{n}^{\mathrm{o}}$. 6119-08-CEEA), determinados pela Câmara de Ética em Experimentação Animal da Universidade Estadual Paulista (FMVZ), Unesp, Jaboticabal/SP, Brasil.

Utilizaram-se 384 suínos (fêmeas e machos castrados) de cruzamento industrial, com peso médio de $115 \mathrm{~kg}$ e provenientes de 16 granjas comerciais. Os animais foram submetidos a um jejum de 12 horas na granja e embarcados com o auxílio de tábuas de manejo. Foram utilizados caminhões, com carroceria metálica de dois pisos, contendo seis divisórias em cada andar. Dois animais, selecionados de forma aleatória em cada divisória, foram identificados com brincos no embarque para posterior avaliação das variáveis de interesse.

O experimento foi delineado em um arranjo fatorial $2 \times 4$, com duas classificações de distância da granja até o frigorífico (mais de $50 \mathrm{~km}$ ou menos de $50 \mathrm{~km}$ ) e quatro protocolos de uso de ducha nos animais. Estes consistiram em: não molhar na granja e não molhar no frigorífico (NMG_NMF); não molhar na granja e molhar no frigorífico (NMG_MOF); molhar na granja e não molhar no frigorífico (MOG_NMF); molhar na granja e molhar no frigorífico (MOG_MOF). A ducha foi caracterizada pela aspersão de água sobre os animais, após o embarque e antes do desembarque.

No frigorífico, os suínos foram desembarcados com o auxílio de plataforma móvel e conduzidos até as baias de descanso (110 suínos/baia). Após seis horas de descanso no frigorífico, sob dieta hídrica, os animais foram conduzidos até o restrainer, submetidos à eletronarcose $(700 \mathrm{~V}$; 1,25Amps), imediatamente sangrados na posição horizontal e suspensos ao fim da mesa de sangria. As carcaças foram acondicionadas em câmara fria, com temperaturas variando entre $1^{\circ} \mathrm{C}$ e $4^{\circ} \mathrm{C}$ por 24 horas. Amostras de sangue (10mL) foram colhidas, com auxílio de copos descartáveis, durante a sangria e transferidas para tubos de centrífuga com 10 gotas de heparina sódica (25000 UI/5mL). As amostras foram posteriormente homogeneizadas e submetidas à centrifugação a 3500rpm/10 minutos em temperatura ambiente, em centrífuga portátil modelo Excelsa Baby II da marca Fanem. Alíquotas de $2 \mathrm{~mL}$ do plasma foram transferidas para tubos criogênicos e armazenadas em ultrafreezer $\left(-80^{\circ} \mathrm{C}\right)$ até a execução das análises. A concentração de cortisol foi analisada pelo método de radioimunoensaio (Coat-A-Count ${ }^{\circledR}$ Cortisol Kit, Siemens, Los Angeles, USA) com a dosagem determinada em contador gama (Gama Count Cobra II- PackardTM) e expressa em $\mu \mathrm{g} / \mathrm{dL}$. A atividade enzimática da lactato-oxidase (mmol/L) foi mensurada por comprimento de onda (546nm e 340nn, respectivamente), utilizando-se espectrofotômetro (RA_XTTM, Technicon).

As lesões na pele foram avaliadas visualmente na meia carcaça esquerda, 24 horas após o abate, seguindo a metodologia descrita por INSTITUT... (1996). As medidas de $\mathrm{pH}$ foram tomadas na meia carcaça esquerda, com uso de pHmetro (Hanna, HI 8314), com sensor de compensação de temperatura (Tec 530) e eletrodo de vidro, aos 45 minutos (pHi) e 24 horas post mortem $(\mathrm{pHu})$. Foram realizadas no lombo (músculo Longissimus), entre a $13^{\mathrm{a}}$ e $14^{\mathrm{a}}$ 
costela, perpendicularmente à linha média da meia carcaça, e no pernil (músculo Semimembranosus), com profundidade média de $3,5 \mathrm{~cm}$.

A cor e a perda de água por exsudação da carne foram avaliadas, nos músculos Longissimus $e$ Semimembranosus, 24 horas após o abate. A cor foi tomada (em triplicata) dentro do espaço CIELab, com avaliação da luminosidade ( $\left.\mathrm{L}^{*}\right)$, da intensidade da cor vermelha $\left(\mathrm{a}^{*}\right)$ e da intensidade da cor amarela (b*), após um período de 30 minutos de exposição das amostras ao oxigênio atmosférico. Utilizou-se o colorímetro Konica Minolta (DL65), ângulo de visão de $0^{\circ}$, com iluminação difusa e componente especular, modelo CR 400, Minolta (Câmera Co., Ltd. Osaka, Japão). Para avaliação da perda de água por exsudação, foram utilizadas amostras de 100 gramas (em duplicatas) de cada músculo. A perda foi determinada pelo método EZ-DripLoss (Rasmussen e Anderson, 1996), utilizando-se recipientes para suco de carne "Meat juice containers" (KABE Labortechnik, NümbrechtElsenroth, Alemanha). Após 48 horas de resfriamento, cada amostra foi pesada novamente. A porcentagem de perda de água foi calculada pela diferença entre o peso inicial e o peso final da amostra dividido pelo peso inicial e multiplicado por 100 (Honikel, 1998).

As perdas por cocção foram calculadas pela diferença de peso (expressa em porcentagem) antes e depois de as amostras serem cozidas em banho-maria. Para isso, quatro amostras de $2,5 \mathrm{~cm}$ de espessura do lombo de cada suíno foram embaladas e congeladas $\left(-20^{\circ} \mathrm{C}\right)$. Posteriormente, foram descongeladas em refrigerador a $5^{\circ} \mathrm{C}$, (24 horas) e divididas em duas subamostras de $100 \mathrm{~g} \quad( \pm 0,05 \mathrm{~g})$. Essas amostras foram embaladas a vácuo, cozidas em banho-maria $\left(80^{\circ} \mathrm{C}\right.$ durante uma hora), colocadas sobre papel absorvente até chegarem à temperatura ambiente e pesadas novamente (Honikel, 1987).

Para a avaliação da maciez, foi utilizado o texturômetro TA XT-Plus Texture Analyser 2i, equipado com dispositivo Warner-Bratzler, com velocidade de descida de $200 \mathrm{~mm} / \mathrm{min}$ (American Meat Science Association, 1995). Cinco cubos com $1 \times 1 \times 2 \mathrm{~cm}$, provenientes de cada amostra da análise de perda de água por cocção, foram colocados com as fibras orientadas no sentido perpendicular às lâminas do aparelho WarnerBlatzler. A média de força (Kgf) necessária para cortar os cinco cubos foi utilizada como valor final.

A carne foi classificada qualitativamente em PSE (Pale, Soft, Exudative), RSE (Red, Soft, Exudative), RFN (Red, Firm, Non-exudative), DFD (Dark, Firm, Dry) ou Pálida por meio da metodologia de Van Heugten (2001) modificada por Araújo (2009).

Para as análises estatísticas, foram gerados blocos por meio da combinação fatorial granjas $\mathrm{x}$ divisão da carroceria, com o fator bloco aninhado dentro de distância, o que resultou em 24 blocos (repetições) para cada um dos tratamentos. Os dados foram submetidos a análises pelo pacote estatístico SAS (Statistical, 2003), utilizando-se modelo com delineamento em blocos ao acaso, com os oito tratamentos dispostos num esquema fatorial $2 \times 4$. Foram testados os efeitos de distância da granja ao frigorífico, de ocorrência ou não de ducha na granja e no frigorífico e a interação entre esses fatores, além do efeito do tratamento molha ou não molha dentro de distância da granja ao frigorífico. Para as variáveis contínuas e de contagem, utilizou-se o procedimento GLM (Statistical..., 2013), e as diferenças entre médias foram testadas por teste de comparações múltiplas ( $\mathrm{t}$ de Student), com nível de significância de 5\%. As variáveis categóricas foram submetidas à análise de frequências e ao teste da razão de verossimilhança (TRV). Quando significativo $(\mathrm{P} \leq 0,05)$, utilizaram-se comparações dos contrastes aos pares por TRV.

\section{RESULTADOS E DISCUSSÃO}

$\mathrm{O}$ uso de ducha não teve influência significativa $(\mathrm{P}>0,05)$ sobre os níveis plasmáticos de cortisol e de lactato. As médias para a concentração de cortisol dos suínos foram NMG_NMF = $10,2 \pm 0,5, \mathrm{NMG} \_\mathrm{MOF}=9,7 \pm 0,4, \mathrm{MOG} \_\mathrm{MOF}=$ $8,7 \pm 0,4$ e MOG_NMF $=9,4 \pm 0,4$. Para o lactato, as médias foram: NMG-NMF $=11,9 \pm 0,5$, NMG_MOF $=11.4 \pm 0,6$, MOG_MOF $=11,0 \pm 0,5$ e MOG_NMF $=12,0 \pm 0,6$. Os valores médios para o cortisol estavam bem abaixo dos encontrados por Brown et al. (1998), ao avaliarem animais manejados em situação comercial. A concentração de lactato também foi próxima ou abaixo da encontrada para animais 
manejados sob condições de mínimo estresse (Hambrecht et al., 2005). Dessa forma, os resultados encontrados devem-se ao tempo de espera no frigorífico, que promoveu o descanso e a recuperação fisiológica dos suínos. Isso pode ter nivelado o resultado dessas variáveis entre todos os tratamentos.

O número de lesões por manejo, densidade ou brigas, assim como o total de lesões na carcaça, também não diferiu significativamente $(\mathrm{P}>0,05)$ entre os tratamentos, independentemente da distância da granja até o frigorífico. Isso indica que o uso da ducha também não teve efeito sobre o comportamento de briga e a facilidade de manejo apresentados pelos animais durante o período pré-abate. De fato, Weeding et al. (1993) não observaram redução no número de briga em suínos pulverizados com água durante a espera no frigorífico. Nos suínos, as brigas e o manejo são algumas das principais causas de estresse (Terlouw et al., 2008). Dessa forma, esses resultados avigoram os encontrados para as variáveis fisiológicas.

Nenhum dos tratamentos testados teve efeito significativo $(\mathrm{P}>0,05)$ sobre o $\mathrm{pH}$ inicial $\left(\mathrm{pH}_{\mathrm{i}}\right)$ e o $\mathrm{pH}$ final $\left(\mathrm{pH}_{\mathrm{u}}\right)$ dos dois músculos avaliados. As médias para o lombo e o pernil foram, respectivamente, 6,37 e 6,40 $( \pm 0,02)$ para $\mathrm{pH}_{\mathrm{i}}$, 5,63 e 5,67 $( \pm 0,01)$ para $\mathrm{pH}_{\mathrm{u}}$. Resultados semelhantes a esses foram reportados por Colleu e Chevillon, (1999). Esses autores não verificaram diferenças para essas variáveis quando utilizaram um banho de cerca de cinco minutos após o embarque dos suínos.

No entanto, o uso de aspersão nas baias de espera logo antes do abate foi eficiente para desacelerar a taxa de queda do pH (Long e Tarrant, 1990) e resultou em carne com menor $\mathrm{pH}$ final (Weeding et al., 1993). Segundo os últimos autores, esse resultado foi provocado pelo efeito do arrefecimento provocado pela água, já que não houve redução no número de brigas quando o recurso foi utilizado. Além disso, Lambooy e Engel (1991) verificaram que o uso de ducha durante o transporte, em temperaturas acima de $20^{\circ} \mathrm{C}$, não teve efeito sobre o $\mathrm{pH}$ do lombo e do pernil quando um sistema de ventilação regulável foi utilizado no caminhão.

O uso da ducha não teve influência significativa $(\mathrm{P}>0,05)$ sobre todas as variáveis da cor do lombo e sobre a luminosidade e o teor de amarelo do pernil. Apenas o teor de vermelho do pernil teve efeito significativo $(\mathrm{P}<0,05)$ do uso da ducha. Os animais do tratamento MOG_MOF apresentaram maior média para essa variável $(5,23)$ quando comparados aos dos demais tratamentos (4,98 para NMG_MOF, 4,93 MOG_NMF e 4,86 para o tratamento controle). Entretanto, essa diferença não foi relevante para diferenciação da qualidade da carne. Isso porque a diferença numérica para essa variável foi pequena entre os tratamentos. Além disso, os resultados para o teor de amarelo e de luminosidade do pernil de todas as variáveis de cor do lombo e de pHi e pHu do lombo e do pernil, indicam que não houve diferenças organolépticas da carne entre os diferentes tratamentos.

A variável perda por cocção sofreu efeito significativo de interação $(\mathrm{P}<0,05)$ entre os fatores, com os tratamentos de molha ou não molha diferindo apenas nas granjas localizadas a mais de $50 \mathrm{~km}$ do frigorífico (Tab. 1). Esse resultado aponta para uma interação entre $o$ efeito da ducha e outros fatores envolvidos no manejo pré-abate. Isso porque, além da distância da viagem, as classificações: longe ou perto, caracterizam também o efeito de sistemas de produção levemente distintos (com possíveis diferenças na genética, alimentação, sistema de cria e preparação para o transporte). No entanto, a diferença encontrada também não representa impacto significativo para a qualidade da carne.

Tabela 1. Médias ( \pm e.p.) para perda por cocção da carne de suínos

\begin{tabular}{lcccc} 
& \multicolumn{4}{c}{ Tratamento $^{1}$} \\
\cline { 2 - 5 } & Controle & NMG_MOF & MOG_MOF & MOG_NMF \\
\hline Perto & $39,0 \pm 0,4$ & $38,5 \pm 0,5$ & $38,4 \pm 0,5$ & $38,2 \pm 0,3$ \\
Longe & $37,0 \pm 0,6 \mathrm{~b}$ & $37,1 \pm 0,4 \mathrm{~b}$ & $37,7 \pm 0,3 \mathrm{ab}$ & $38,2 \pm 0,3 \mathrm{a}$ \\
\hline
\end{tabular}

${ }^{1}$ Controle $=$ não molhados na granja e não molhados no frigorífico; NMG_MOF = não molhados na granja e molhados no frigorífico; MOG_MOF = molhados na granja e molhados no frigorífico; $M O G \_N M F=$ molhados na granja e não molhados no frigorífico.

Médias seguidas de mesma letra não diferem entre si pelo teste da razão de verossimilhança $(\mathrm{P}<0,05)$. 
$O$ uso de ducha não teve efeito significativo $(\mathrm{P}>0,05)$ sobre a classificação qualitativa da carne. Apenas o tratamento MOG_MOF apresentou carnes classificada como PSE ou DFD (1\% para cara classificação), ambas consideradas como não desejáveis pela indústria e pelo consumidor. Contudo, isso corresponde à apenas um animal para cada classificação, não tendo efeito estatístico significativo. Os animais que apresentaram carne Pálida e RSE somaram $21,7 \%$ para o tratamento MNG_NMF e 18,2 para MOG_NMF, 29,7\% para NMG_MOF e 28,4\% para MOG_MOF, diferença não significativa estatisticamente. Dessa forma, o uso da ducha também não foi eficiente para alterar a classificação qualitativa da carne dos suínos.

A perda por gotejamento do pernil não foi significativamente influenciada $(\mathrm{P}>0,05)$ pelo uso da ducha e pela distância da viagem. Todas as demais variáveis relacionadas à qualidade da carne sofreram efeito $(\mathrm{P}<0,05)$ apenas da distância da granja ao frigorífico. Os animais oriundos de granjas localizadas a mais de $50 \mathrm{~km}$ apresentaram carne com maior força de cisalhamento, menor luminosidade, menor teor de vermelho e maior teor de amarelo (Tab. 2). A média para perda por gotejamento do lombo dos animais provenientes dessas granjas também foi menor, quando comparada à de animais provenientes de granjas localizadas a menos de $50 \mathrm{~km}$ do frigorífico (Tab. 1). Porém, apesar dessa diferença, a média dessa variável para ambas as distâncias da granja ao frigorífico ficou abaixo de 5\%, índice considerado como desejável (Kauffman, 1991). Além disso, a verificação de diferenças significativas entre as distâncias de viagem apenas para algumas características da cor e da perda de água da carne, e não para o nível de cortisol e lactato, indica que essas distinções provavelmente se devem a outros fatores, como: diferenças de genética e sistema de criação dos animais que compõem esses grupos, e não necessariamente aos efeitos do transporte.

Tabela 2. Valores médios ( \pm e.p.) dos indicadores relacionados à qualidade da carne de suínos de acordo com a distância da viagem ao frigorífico

\begin{tabular}{lcc}
\multicolumn{1}{c}{ Variável } & \multicolumn{2}{c}{ Distância da granja ao frigorífico } \\
\cline { 2 - 3 } & $<50 \mathrm{~km}$ & $>50 \mathrm{~km}$ \\
\hline Luminosidade do lombo & $44,3 \pm 0,3 \mathrm{a}$ & $38,7 \pm 0,3 \mathrm{~b}$ \\
Teor de vermelho do lombo & $4,66 \pm 0,1 \mathrm{a}$ & $3,35 \pm 0,1 \mathrm{~b}$ \\
Teor de amarelo do lombo & $-2,27 \pm 0,1 \mathrm{~b}$ & $1,99 \pm 0,1 \mathrm{a}$ \\
Luminosidade do pernil & $45,73 \pm 0,3 \mathrm{a}$ & $39,70 \pm 0,3 \mathrm{~b}$ \\
Teor de vermelho do pernil & $5,35 \pm 0,1 \mathrm{a}$ & $4,65 \pm 0,1 \mathrm{~b}$ \\
Teor de amarelo do pernil & $-1,56 \pm 0,1 \mathrm{~b}$ & $-1,18 \pm 0,1 \mathrm{a}$ \\
Perda por gotejamento do lombo, $\%$ & $4,06 \pm 0,2 \mathrm{a}$ & $3,58 \pm 0,2 \mathrm{~b}$ \\
Força de cisalhamento, Kgf & $7,6 \pm 0,2 \mathrm{~b}$ & $9,4 \pm 0,7 \mathrm{a}$ \\
\hline
\end{tabular}

Médias seguidas de mesma letra não diferem entre si pelo teste da razão de verossimilhança $(\mathrm{P}<0,05)$.

A distância da granja ao frigorífico também teve efeito $(\mathrm{P}<0,001)$ sobre a classificação de qualidade da carne. O número de animais que apresentaram carne classificada como Pálida foi $10,11 \%$ maior entre os provenientes de granjas localizadas perto do que entre os oriundos de granjas longe do frigorífico. Os animais classificados com carne PSE e DFD representaram menos de $1 \%$ nas duas situações.

Durante o período pré-abate, os animais podem sofrer estresse psicológico, devido às mudanças do ambiente, a distúrbios sociais e à manipulação, bem como estresse físico, devido à privação de alimentos, a condições climáticas, à fadiga e, por vezes, à dor (Terlouw et al., 2008). As interações entre esses muitos fatores pode ter dificultado a interpretação do verdadeiro efeito da ducha sobre a concentração de lactato e cortisol, o número de lesões na pele e os parâmetros de qualidade da carne. Assim, o efeito de relaxamento e arrefecimento provocado pelo banho pode não ser percebido ou eficiente para provocar mudanças nessas variáveis, quando os animais estão submetidos a muitos estímulos estressantes, como normalmente acontece no período pré-abate. Diferenças entre grupos molhados ou não molhados talvez possam 
ser percebidas quando todos os demais fatores forem controlados. Entretanto, esse controle não é possível de ser realizado em situações comerciais, como as avaliadas aqui.

Além disso, quando esse experimento foi realizado, a legislação brasileira não permitia períodos de descanso no frigorífico menores que seis horas. Dessa forma, o longo período de descaso utilizado aqui provavelmente mascarou os efeitos do uso da ducha sobre as variáveis fisiológicas e de qualidade da carne. Atualmente, períodos de descanso mais curtos são permitidos, e o efeito da ducha nessas condições pode ser melhor estudado.

\section{CONCLUSÃO}

Em situações comerciais, com um tempo longo de descanso no frigorífico, o uso de ducha, antes e/ou após o transporte para o abate, não tem influência sobre o bem-estar animal e a qualidade da carne e da carcaça de suínos. Portanto, outros métodos de arrefecimento devem ser estudados e implantados nessa situação, o que evitaria o trabalho desnecessário, contribuiria para a economia de água no processo produtivo e a redução do risco de poluição. Atualmente, esses pontos ganham destaque, uma vez que um dos focos da suinocultura moderna é a mitigação dos efeitos da atividade produtora no meio ambiente. Outras pesquisas com o acompanhamento de variáveis fisiológicas, relacionadas ao estresse e à termorregulação dos animais durante todo o período pré-abate e com menores períodos de descanso no frigorífico, devem ser realizadas, a fim de auxiliar nas recomendações de uso dessa técnica.

\section{REFERÊNCIAS}

ARAÚJO, A.P. Manejo pré-abate e bem-estar dos suínos em frigoríficos brasileiros. 2009. 123f. Dissertação (Mestrado) - Faculdade de Medicina Veterinária e Zootecnia, Universidade Estadual Paulista Júlio de Mesquita Filho, Botucatu.

AMERICAN Meat Science Association. Research guideliness for cookery sensory and instrumental tenderness measurement of fresh meat. Chicago: AMSA, 1995. 48p.
BROWN, S.N.; WARRISS, P.D.; NUTE, G.R. et al. Meat quality in pigs subjected to minimal preslaughter stress. Meat Sci., v.49, p.257-265, 1998.

COLLEU, T.; CHEVILLON, P. Intérêt pour la qualité de la viande et le bien-être, du douchage dans le camion des porcs à l'embarquement. Techni-porc, v.22, p.23-30, 1999.

DALLA COSTA, O.A.; LUDKE, J.V.; COSTA, M.J.R.P. et al. Efeito das condições pré-abate sobre a qualidade da carne de suínos pesados. Arch. Zootec., v.59, p.391-402, 2010.

FAUCITANO, L.; CHEVILLON, P.; ELLIS, M. Effects of feed withdrawal prior to slaughter and nutrition on stomach weight, and carcass and meat quality in pigs. Livest. Sci., v.127, p.110114, 2010.

GREGORY, N.G. Animal welfare at markets and during transport and slaughter. Meat Sci., v.80, p.2-11, 2008.

HAMBRECHT, E.; EISSEN, J.J.; NOOIJENT, R.I. et al. Negative effects of stress immediately before slaughter on pork quality are aggravated by suboptimal transport and lairage conditions. $J$. Anim. Sci., v.83, p.440-448, 2005.

HONIKEL K.O. Influence of chilling on meat quality attributes of fast glycolysing pork muscles. In: TARRANT, P.V.; EIKELENBOOM, G.; MONIN, G. Evaluation and control of meat quality in pigs. Dordrecht: Martinius Nijhoff, 1987. p.273-283.

HONIKEL, K.O. Reference methods for the assessment of physical characteristics of meat. Meat Sci., v.49, p.447-457, 1998.

INSTITUT Technique Du Porc. Notation des hématomes sur couenne - porcs vivant ou carcasses. Le Rheu: ITP, 1996. 45p.

KAUFFMAN, R.G. Electronic evaluation of meat quality. In: SYMPOSIUM ELECTRONIC EVALUATION OF MEAT IN SUPPORT OF VALUE-BASED MARKETING, 1991, Indiana. Proceedings... Indiana: Purdue University, 1991. p.199-221.

LAMBOOY, E.; ENGEL B. Transport of slaughter pigs by truck over a long-distance some aspects of loading density and ventilation. Livest. Prod. Sci., v.28, p.163-174, 1991. 
LONG, V.P.; TARRANT, P.V. The effect of pre-slaughter showering and post-slaughter rapid chilling on meat quality in intact pork sides. Meat Sci., v.27, p.181-195, 1990.

MOTA-ROJAS, D.; OROZCO-GREGORIO, H.; GONZALEZ-LOZANO, M. et al. Therapeutic approaches in animals to reduce the impact of stress during transport to the slaughterhouse: a review. Int. J. Pharmacol., v.7, p.568-578, 2011.

RASMUSSEN, A.J.; ANDERSON, M. New method for determination of drip loss in pork muscles. In: INTERNATIONAL CONGRESS OF MEAT SCIENCE AND TECHNOLOGY, 42., 1996, Lillehammer. Proceedings... Lillehammer: Noeway, 1996, p.286-287.
STATISTICAL Analysis System Institute SAS, 2003. System for Microsoft windows, release 9.1. 1 CD-Rom.

TERLOUW, E.M.C.; ARNOULD, C.; AUPERIN, B. et al. Pre-slaughter conditions, animal stress and welfare: current status and possible future research. Animal, v.2, p.15011517, 2008.

VAN HEUGTEN, E. Understanding pork quality. Swine News, v.24, p.1-4, 2001.

WEEDING, C.M.; GUISE, H.J.; PENNY, R.H.C. Factors influencing the welfare and carcass and meat quality of pigs: the use of water sprays in lairage. Anim. Prod., v.56, p.393-397, 1993. 